\title{
On Partial Charge Transfer Processes in Multiparticle Systems on Graphs
}

\author{
Victor Chulaevsky \\ Département De Mathématiques, Université De Reims, Moulin De La Housse, B.P. 1039, 51687 Cedex 2 Reims, France \\ Correspondence should be addressed to Victor Chulaevsky; plinius@free.fr
}

Received 28 June 2014; Accepted 16 September 2014; Published 21 October 2014

Academic Editor: Lingju Kong

Copyright ( 2014 Victor Chulaevsky. This is an open access article distributed under the Creative Commons Attribution License, which permits unrestricted use, distribution, and reproduction in any medium, provided the original work is properly cited.

\begin{abstract}
We assess the probability of resonances between sufficiently distant states of an $N$-particle disordered quantum system in a combinatorial graph $\mathscr{Z}$. In the $N$-particle configuration space, there are arbitrarily distant pairs of configurations giving rise to pairs of local (random) Hamiltonians which are strongly coupled, so that the eigenvalue concentration (EVC) bounds are difficult to obtain. We extend to any number of particles the efficient EVC bounds, obtained earlier for the 2-particle systems.
\end{abstract}

\section{Introduction}

We study quantum systems in a disordered environment, usually referred to as Anderson-type models, due to the seminal paper by Anderson [1]. For nearly fifty years following its publication, the localization phenomena have been studied in the single-particle approximation, that is, under the assumption that the interaction between particles subject to the common random external potential is sufficiently weak to be neglected in the analysis of the decay properties of eigenstates of the multiparticle system in question. A detailed discussion of recent developments in the physics of disordered media is most certainly beyond the scope of this paper; we simply refer to the papers by Basko et al. [2] and by Gornyi et al. [3] where it was shown, in the framework of physical models and methods, that the localization phenomena, firmly established for the noninteracting systems, persist in presence of nontrivial interactions.

The mathematical Anderson localization theory has motivated a large number of studies of random differential and finite-difference operators during the last forty years, but only recently a significant progress has been made in the rigorous theory of multiparticle quantum systems in a disordered environment with a nontrivial interparticle interaction (cf. [4-6] and more recent works [7-10]), and there still remain many challenging open problems in this area of mathematical physics. Such problems are often related to the EVC bounds, which in the single-particle setting go back to the celebrated Wegner bound [11]. Building on our results from [12], we discuss in the present paper some important implications for the Hamiltonians of the multiparticle disordered quantum systems.

Specifically, we consider a system of $N \geq 2$ quantum particles in a finite or countable, locally finite connected graph $\mathscr{Z}$, endowed with the canonical graph distance $d(\cdot, \cdot)=$ $d_{\mathscr{I}}(\cdot, \cdot)$, with the Hamiltonian of the following form:

$$
\mathbf{H}=\sum_{j=1}^{N}\left(\Delta^{(j)}+V\left(x_{j}, \omega\right)\right)+\mathbf{U}
$$

where $V: \mathscr{Z} \times \Omega \rightarrow \mathbb{R}$ is a random field on the graph $\mathscr{Z}$, relative to a probability space $(\Omega, \mathscr{F}, \mathbb{P}), \Delta^{(j)}$ is the graph Laplacian on $\mathscr{E}$, namely:

$$
\begin{aligned}
\Delta^{(j)} & \Psi\left(x_{1}, \ldots, x_{N}\right) \\
& =\sum_{y \in \mathbb{Z}^{d}: d\left(y, x_{j}\right)=1} \Psi\left(x_{1}, \ldots, x_{j-1}, y, \ldots, x_{N}\right),
\end{aligned}
$$

and the interaction operator $\mathbf{U}$ is the multiplication by a function $\mathbf{U}(\mathbf{x})$ which we assume to be bounded (this assumption can be relaxed). The symmetry of the function $\mathbf{U}$ is not required, and we do not assume $\mathbf{U}$ to be a "shortrange" or rapidly decaying interaction. In fact, we focus 
here on the restrictions of $\mathbf{H}$ to bounded subsets of the $N$ particle configuration space. The decay rate of the interaction is important for the localization analysis.

Our main assumptions on the random field $V$ are presented in Section 1.3 (cf. (14)). See also Theorem 4 (cf. [12], Theorem 4) which describes explicitly a class of IID random fields satisfying the property (14).

1.1. Eigenvalue Concentration Bounds. We focus on the probabilistic eigenvalue concentration bounds, known as the Wegner-type bounds, due to the celebrated paper by Wegner [11]. It would not be an exaggeration to say that this bound is the heart of the multiscale analysis (MSA, cf. [13]). In a slightly disguised form, it also appears in the framework of the fractional-moment method (FMM) both in its singleparticle and in its multiparticle versions, as the reader can observe in $[6,14]$. In essence, a Wegner-type bound is a probabilistic bound of the following form:

$$
\mathbb{P}\left\{\operatorname{dist}\left(E, \sigma\left(\mathbf{H}_{\Lambda}(\omega)\right)\right) \leq \epsilon\right\} \leq f(|\Lambda|, \epsilon),
$$

where $\mathbf{H}_{\Lambda}(\omega)$ is the restriction of $\mathbf{H}(\omega)$ on a bounded subset $\Lambda$ with some self-adjoint boundary conditions and $\sigma\left(\mathbf{H}_{\Lambda}(\omega)\right)$ is its spectrum (a finite number of random points, in the case of lattice models).

The role and importance of such bounds can be easily understood: the MSA procedure starts with the analysis of the resolvents $\left(\mathbf{H}_{\Lambda}-E\right)^{-1}$, so it is vital to know how unlikely it is to have the spectrum of $\mathbf{H}_{\Lambda}$ close to $E \in \mathbb{R}$.

We introduce the graph structure in the configuration space $\mathscr{Z}^{N}$ of $N$ distinguishable particles in Section 2.1. Simply put, we consider the product metric space $(\mathscr{Z})^{N}$ with the max-distance $\rho(\cdot, \cdot)$ (cf. (22)). Given any finite ball $\mathbf{B}_{L}(\mathbf{u}):=\left\{\mathbf{x} \in \mathscr{Z}^{N} \mid \boldsymbol{\rho}_{\mathscr{X}}(\mathbf{x}, \mathbf{u}) \leq L\right\}$, we will consider a finitevolume approximation of the following operator:

$$
\mathbf{H}_{\mathbf{B}_{L}(\mathbf{u})}=\mathbf{1}_{\mathbf{B}_{L}(\mathbf{u})} \mathbf{H} \mathbf{1}_{\mathbf{B}_{L}(\mathbf{u})} \Upsilon_{\ell^{2}\left(\mathbf{B}_{L}(\mathbf{u})\right)},
$$

acting in the finite-dimensional Hilbert space $\ell^{2}\left(\mathbf{B}_{L}(\mathbf{u})\right)$.

In [15], where the 1-particle configuration space $\mathscr{Z}$ was assumed to be $\mathbb{Z}^{d}$, the following "two-volume" version of the Wegner bound was established for the pairs of two-particle operators $\mathbf{H}_{\mathbf{B}_{L}(\mathbf{u})}$ and $\mathbf{H}_{\mathrm{B}_{L^{\prime}}\left(\mathbf{u}^{\prime}\right)}$ with $L \geq L^{\prime}$ and such that $\operatorname{dist}\left(\mathbf{B}_{L}(\mathbf{u}), \mathbf{B}_{L^{\prime}}\left(\mathbf{u}^{\prime}\right)\right)>8 L$ : if $v$ is the continuity modulus of the marginal distribution of the IID random field $V$, then

$$
\begin{gathered}
\mathbb{P}\left\{\operatorname{dist}\left(\sigma\left(\mathbf{H}_{\mathbf{B}_{L}(\mathbf{u})}\right), \sigma\left(\mathbf{H}_{\mathbf{B}_{L^{\prime}}\left(\mathbf{u}^{\prime}\right)}\right)\right) \leq \epsilon\right\} \\
\leq(2 L+1)^{2 d}\left(2 L^{\prime}+1\right)^{d} v(2 \epsilon) .
\end{gathered}
$$

The proof given in [15] is based on a geometrical notion of "separable" pairs of balls, combined with Stollmann's lemma on diagonally monotone functions. In [16] a similar bound was proven in the case of IID random field $V$ with analytic marginal distribution.

Starting from $N=3$, additional difficulties appear in the analysis of pairs of spectra $\sigma\left(\mathbf{H}_{\mathbf{B}_{L}(\mathbf{u})}\right)$ and $\sigma\left(\mathbf{H}_{\mathbf{B}_{L}\left(\mathbf{u}^{\prime}\right)}\right)$. To put it simply, no priori lower bound on the distance
$\operatorname{dist}\left(\mathbf{B}_{L}(\mathbf{u}), \mathbf{B}_{L}\left(\mathbf{u}^{\prime}\right)\right)>C L$ between two balls of radius $O(L)$ can guarantee the approach of [15] to work, no matter how large the constant $C$ is. This gives rise to a significantly more sophisticated scaling procedure for $N \geq 3$. A similar difficulty arises in [6].

1.2. The Main Goal. In $[4,5]$, a multiparticle adaptation of the MSA was used to prove spectral localization (i.e., exponential decay of eigenfunctions) in the strong disorder regime. Aizenman and Warzel [6] used the FMM to prove directly dynamical localization (hence, spectral localization) in various parameter regions including strong disorder and weak interactions.

Despite many differences between the MSA and the FMM, similar technical difficulties have been encountered in both cycles of papers. Namely, it turned out to be difficult to prove the decay bounds of eigenfunctions $\Psi_{j}^{(N)}\left(x_{1}, \ldots, x_{n}\right)$ of $N$-particle Hamiltonians in terms of some norm $\|\cdot\|$ in $\mathbb{R}^{N d} \supset \mathbb{Z}^{N d}$ (recall that the configuration space in $[5,6]$ was the lattice $\left.\left(\mathbb{Z}^{d}\right)^{N}\right)$ :

$$
\left|\Psi_{j}^{(N)}\left(x_{1}, \ldots, x_{n} ; \omega\right)\right| \leq C_{j}(\omega) e^{-m\|\mathbf{x}\|} .
$$

If the interaction $\mathbf{U}$ is symmetric (and so is, then, $\mathbf{U}+\mathbf{V}$ ), then it is natural to expect (or to fear...) "resonances", and "tunneling" processes, between a point $\mathbf{x}=\left(x_{1}, \ldots, x_{N}\right)$ and the points $\tau(\mathbf{x})=\left(x_{\tau(1)}, \ldots, x_{\tau(N)}\right)$ obtained by permutations $\tau \in \mathfrak{S}_{N}$. In this context, it is much more natural to use the following symmetrized distance:

$$
d_{S}(\mathbf{x}, \mathbf{y}):=\min _{\tau \in \overleftarrow{S}_{N}}\|\tau(\mathbf{x})-\mathbf{y}\| .
$$

(Recall that, for the moment, we are discussing the papers [5, 6] where $\mathscr{Z}=\mathbb{Z}^{d}$, so the distance in $\mathscr{Z}$ can be defined with the help of a norm.) Note also that if the quantum particles are bosons or fermions, then all the points $\tau(\mathbf{x}), \tau \in \mathbb{S}_{N}$, should even be considered as identical, or, more precisely, the spectral problem should be solved in the subspace of symmetric or antisymmetric functions of variables $x_{j}$.

However, due to a highly correlated nature of the potential of a multiparticle system, even the above concession did not suffice, and it was easier to use the "Hausdorff distance" (which is a pseudometric) between the points $\mathbf{x}, \mathbf{y} \in \mathscr{Z}^{N}$. This resulted in substantally weaker decay estimates than expected.

Aizenman and Warzel [6] analyzed the aforementioned technical problem and pointed out that, physically speaking, it was difficult to rule out the possibility of "tunneling" between the points $\mathbf{x}$ and $\mathbf{y}$ related by a "partial charge transfer" process, for example, between the points $(a, a, b)$ and $(a, b, b), a \neq b$, corresponding to the following states:

state $\mathbf{x}: 2$ particles at the point $a$ and 1 particle at $b$,

state $\mathbf{y}: 1$ particle at the point $a$ and 2 particles at $b$.

Observe that the norm-distance between such states can be arbitrarily large. What is even more important for the 
applications to physics is that the uncontrollable pairs of loci in the configuration space, which may (or might) give rise to quantum tunneling over large distances, occur in any finite domain of the physical space, no matter how large it is.

As a result, one could not prove complete localization, say, in a finite cube $[-L, L]^{d}, L \gg 1$. Surprisingly, here the localization analysis of an actually infinite system turned out to be substantially simpler than for its finite-size counterpart.

In the present paper we address this problem and show that abnormally strong resonances between distant states in the configuration space, related by partial charge transfer processes, are unlikely and prove efficient probabilistic estimates for such unlikely situations.

1.3. The Main Result. In this paper, we work with connected, locally finite graphs $(\mathscr{Z}, \mathscr{E})$ with the vertex set $\mathscr{Z}$ and the edge set $\mathscr{E}$; by slight abuse of notations, we often identify the graph with $\mathscr{Z}$.

We assume that the growth rate of the balls in $\mathscr{Z}$ is uniformly bounded, namely:

$$
\sup _{x \in \mathscr{E}}\left|B_{L}(x)\right| \leq f_{\mathscr{X}}(L)<+\infty .
$$

While a significant part of the techniques and intermediate results in our paper does not rely, formally, upon any particular upper bound on the cardinality of the balls, the main application (to the multiscale analysis) requires a subexponential bound on $f_{\mathscr{X}}$.

We consider in particular the class $\mathfrak{Z}\left(d, C_{d}\right)$ of polynomially growing graphs: $\mathscr{Z} \in \mathcal{Z}\left(d, C_{d}\right)$ if, for some $d, C_{d} \in$ $(0,+\infty)$, the following bound holds:

$$
f_{\mathscr{X}}(L)=\sup _{x \in \mathscr{E}}\left|B_{L}(x)\right| \leq C_{d} L^{d}, \quad L \geq 1 .
$$

The class of graphs with $f_{\mathscr{L}}(L) \leq$ Const $e^{L^{\delta}}, 0<\delta<1$, is also suitable for the multiscale analysis, but actually the most interesting case after the lattices $\mathbb{Z}^{d}$ are the trees and other graphs with exponential growth rate of balls, and the latter, as is well-known, remains so far beyond the reach of the MSA techniques.

We assume that an IID random field $V: \mathscr{Z} \times \Omega \rightarrow \mathbb{R}$, relative to a probability space $(\Omega, \mathfrak{F}, \mathbb{P})$, is defined on $\mathscr{Z}$.

Introduce the following notations. Given a finite subset $Q \subset \mathscr{Z}$ (of cardinality $|Q| \equiv$ cardQ), we denote by $\xi_{Q}(\omega)$ the sample mean of the random field $V$ over $Q$ as follows:

$$
\xi_{Q}(\omega)=\frac{1}{|Q|} \sum_{x \in Q} V(x, \omega)
$$

and we define the fluctuations of $V$ relative to $\xi_{Q}(\omega)$ as follows:

$$
\eta_{x}(\omega)=V(x, \omega)-\xi_{\mathrm{Q}}(\omega), \quad x \in \mathrm{Q} .
$$

Let $\mathfrak{F}_{\mathrm{Q}}$ be the sigma-algebra generated by $\left\{\eta_{x}, x \in \mathrm{Q}\right\}$ and $F_{\xi}\left(\cdot \mid \mathfrak{F}_{Q}\right)$ be the conditional probability distribution function of $\xi_{\mathrm{Q}}$ given $\mathfrak{\Im}_{\mathrm{Q}}$ :

$$
F_{\xi}\left(s \mid \mathfrak{F}_{Q}\right):=\mathbb{P}\left\{\xi_{Q} \leq s \mid \mathfrak{F}_{Q}\right\} .
$$

For a given $s \in \mathbb{R}, F_{\xi}\left(s \mid \mathfrak{F}_{\mathrm{Q}}\right)$ is a random variable, determined by the values of $\left\{\eta_{x}, x \in Q\right\}$, but we will often use inequalities involving it, meaning that these relations hold true for $\mathbb{P}$-a.e. condition.

We will assume that the random field $V$ satisfies the following condition (RCM is regularity of the conditional mean).

(RCM): There exist constants $C^{\prime}, C^{\prime \prime}, A^{\prime}, A^{\prime \prime}, b^{\prime}, b^{\prime \prime} \in[0$, $+\infty)$ such that, for any finite subset $Q \subset \mathscr{Z}$, the conditional probability distribution function $F_{\xi}\left(\cdot \mid \mathfrak{F}_{Q}\right)$ satisfies for all $s \in$ $(0,1)$ as follows:

$$
\begin{gathered}
\mathbb{P}\left\{\sup _{t \in \mathbb{R}}\left|F_{\xi}\left(t+s \mid \mathfrak{F}_{Q}\right)-F_{\xi}\left(t \mid \mathfrak{F}_{Q}\right)\right| \geq C^{\prime}|Q|^{A^{\prime}} s^{b^{\prime}}\right\} \\
\leq C^{\prime \prime}|Q|^{A^{\prime \prime}} s^{b^{\prime \prime}} .
\end{gathered}
$$

In fact, for the applications to the MSA, it suffices to require the bound (14) to hold at least for $s \in\left(0, s_{*}\right)$ for some sufficiently small $s_{*}>0$.

Example 1. In the particular case of a Gaussian IID field $V$, for example, with zero mean and unit variance, $\xi_{Q}$ is a Gaussian random variable with variance $|Q|^{-1}$, independent of the fluctuations $\eta_{x}$, so that its probability density is bounded as follows:

$$
p_{\xi_{Q}}(s)=|Q|^{1 / 2}(2 \pi)^{-1 / 2} e^{-|Q| s^{2} / 2} \leq|Q|^{1 / 2}(2 \pi)^{-1 / 2},
$$

although the $L^{\infty}$-norm of its probability density grows as $|Q| \rightarrow \infty$, and so does the continuity modulus of the distribution function $F_{\xi_{Q}}$. In this case, (14) holds deterministically, with $C^{\prime \prime}=0, A^{\prime}=1 / 2$, and $b^{\prime}=1$ as follows:

$$
\mathbb{P}\left\{\sup _{t \in \mathbb{R}}\left|F_{\xi}\left(t+s \mid \mathfrak{F}_{Q}\right)-F_{\xi}\left(t \mid \mathfrak{F}_{Q}\right)\right|>\frac{|Q|^{1 / 2}}{\sqrt{2 \pi}} s\right\}=0 .
$$

In general, the conditional probability distribution function $F_{\xi}\left(\cdot \mid \mathfrak{F}_{Q}\right)$ is not necessarily uniformly continuous, let alone Hölder-continuous. Moreover, the following elementary example shows that for some conditions the distribution of the sample mean can be extremely singular.

Example 2. Let $v_{1}(\omega), v_{2}(\omega)$ be two independent random variables uniformly distributed in $[0,1]$. Set $\xi=\left(v_{1}+v_{2}\right) / 2$ and $\eta=\left(v_{1}-v_{2}\right) / 2$. Conditioning on $\eta \geq 0$ induces a uniform probability distribution on the segment $I(\eta)=\{(t+2 \eta, t), t \in$ $(0,1-2 \eta)\}$ of length $|I(\eta)|=1-2 \eta$, with constant probability density $(1-2 \eta)^{-1}$, if $\eta<1 / 2$. Obviously, these distributions are not uniformly continuous. Moreover, for $\eta=1 / 2, \xi$ takes a single value: $\xi=1 / 2$, so that its conditional distribution is no longer continuous. Observe, however, that the "singular" conditions have probability zero and conditions which give rise to large conditional density of $\xi$ have small probability.

Formally speaking, the condition (RCM) does not refer to the growth rate of balls in the graph $\mathscr{Z}$, but the analytic form of the estimate (14) is in fact adapted to the class $3\left(d, C_{d}\right)$. 
(It can be easily extended to the graphs with sub-exponential growth.) The same remark can be made concerning the formulation of our main result (where we make use of the symmetrized distance $\boldsymbol{\rho}_{S}$ defined in (23)-(22)).

Theorem 3. Let $V: \mathscr{Z} \times \Omega \rightarrow \mathbb{R}$ be a random field satisfying (RCM). Then for any pair of $N$-particle operators $\mathbf{H}_{\mathbf{B}_{L^{\prime}}\left(\mathbf{u}^{\prime}\right)}$ and $\mathbf{H}_{\mathbf{B}_{L^{\prime \prime}}\left(\mathbf{u}^{\prime \prime}\right)}, 0 \leq L^{\prime}, L^{\prime \prime} \leq L$, satisfying $\boldsymbol{\rho}_{S}\left(\mathbf{u}^{\prime}, \mathbf{u}^{\prime \prime}\right)>(4 N-2) L$, and any $s>0$ the following bound holds:

$$
\mathbb{P}\left\{\operatorname{dist}\left(\sigma\left(\mathbf{H}_{\mathbf{B}_{L^{\prime}}\left(\mathbf{u}^{\prime}\right)}\right), \sigma\left(\mathbf{H}_{\mathbf{B}_{L^{\prime \prime}}\left(\mathbf{u}^{\prime \prime}\right)}\right)\right) \leq s\right\}=h_{L}(2 s),
$$

with

$$
h_{L}(s):=\left|\mathbf{B}_{L^{\prime \prime}}(\mathbf{x})\right| \cdot\left|\mathbf{B}_{L^{\prime \prime}}(\mathbf{y})\right| C^{\prime} L^{A^{\prime}} s^{b^{\prime}}+C^{\prime \prime} L^{A^{\prime \prime}} s^{b^{\prime \prime}} .
$$

The following statement establishes the validity of the condition (RCM) for a class of IID random potentials, including the popular in physics uniform distribution in a bounded interval.

Theorem 4 (cf. [12], Theorem 4). Consider an IID random field $V: \mathscr{Z} \times \Omega \rightarrow \mathbb{R}$ admitting a smooth marginal probability density $p_{V}$ with supp $p_{V} \subset[0, \ell]$, satisfying the following conditions:

$$
\begin{gathered}
\forall t \in[0, \ell] \quad 0<p_{V}^{*} \leq p_{V}(t) \leq \bar{p}_{V}<+\infty ; \\
\left\|p_{V}^{\prime} \mathbf{1}_{(0, \ell)}\right\|_{\infty} \leq C_{V}^{\prime}<+\infty .
\end{gathered}
$$

Then $V$ satisfies the condition (RCM) with the following:

$$
\begin{gathered}
C^{\prime}=1, \quad b^{\prime}=0, \quad A^{\prime}=\frac{2}{3}, \\
C^{\prime \prime}=\frac{4 \bar{p}_{V}^{2}}{\ell^{2}}, \quad b^{\prime \prime}=2, \quad A^{\prime}=\frac{2}{3} .
\end{gathered}
$$

\section{Distinguishable Particle Configurations and Weak Separability}

2.1. Basic Definitions. Given a connected graph $(\mathscr{Z}, \mathscr{E})$ and an integer $N \geq 2$, introduce the product graph $\left(\mathscr{E}^{N}, \mathscr{E}^{(N)}\right)$ with the vertex set $\mathscr{Z}^{N} \equiv(\mathscr{Z})^{N}$ and the edge set defined as follows: with $\mathbf{x}=\left(x_{1}, \ldots, x_{N}\right), \mathbf{y}=\left(y_{1}, \ldots, y_{N}\right) \in \mathscr{Z}^{N}$, one has the following condition:

$$
(\mathbf{x}, \mathbf{y}) \in \mathscr{E}^{(N)} \Longleftrightarrow \sum_{j=1}^{N} d_{\mathscr{E}}\left(x_{j}, y_{j}\right)=1 .
$$

Observe that this definition gives the conventional graph structure on the lattice $\mathbb{Z}^{N d}$ considered as the product $\left(\mathbb{Z}^{d}\right)^{N}$, $d \geq 1, N \geq 2$.

Intervals of integer values will often appear in our formulae, and it is convenient to use the standard notation $[[a, b]]:=[a, b] \cap \mathbb{Z}$.

We identify $N$-tuplets $\mathbf{x} \in \mathscr{Z}^{N}$ with configurations of $N$ distinguishable particles in $\mathscr{Z}: \mathbf{x} \equiv\left(x_{1}, \ldots, x_{N}\right) \in \mathscr{Z} \times \cdots \times \mathscr{Z}$.
Pictorially, $\mathbf{y}$ is a nearest graph neighbor of $\mathbf{x}$ in $\mathscr{Z}^{N}$ if it is obtained from $\mathbf{x}$ by moving exactly one particle $x_{j}$ to an adjacent vertex $y_{j} \in \mathscr{Z}$ and vice versa.

The graph structure defines in $\mathscr{Z}^{N}$ the canonical graph distance $\mathbf{d}_{\mathscr{Z}^{N}}$ : for $\mathbf{x} \neq \mathbf{y}, d_{\mathscr{Z}^{N}}(\mathbf{x}, \mathbf{y})$ is the length of the shortest path $\mathbf{x} \leadsto$ y over the edges of $\mathscr{Z}^{N}$. With $\mathscr{Z}$ fixed, we will often drop the subscript and simply write $\mathbf{d}(\mathbf{x}, \mathbf{y})$.

One can also define the max-distance on $\mathscr{Z}^{N}$ :

$$
\boldsymbol{\rho}(\mathbf{x}, \mathbf{y}):=\max _{j \in[[1, N]]} d\left(x_{j}, y_{j}\right) .
$$

It turns out that the graph distance $\mathbf{d}$ and the maxdistance $\rho$ are not well-adapted to the analysis of quantum resonances in the multi particle systems, so we also introduce the symmetrized max-distance by

$$
\rho_{S}(\mathbf{x}, \mathbf{y}):=\min _{\tau \in \Im_{N}} \rho(\tau(\mathbf{x}), \mathbf{y})
$$

The importance of the symmetrized distance can be explained as follows. First, assume that the interaction $\mathbf{U}$ is permutation symmetric; consider the following noninteracting Hamiltonian:

$$
\mathbf{H}^{n i}=\sum_{j=1}^{N}\left(H_{0 ; j}+g V\left(x_{j} ; \omega\right)\right),
$$

being always permutation symmetric; the full Hamiltonian $\mathbf{H}=\mathbf{H}^{n i}+\mathbf{U}$ then also is permutation invariant. Therefore, if $\mathbf{x}=\tau(\mathbf{y})$, for some $\tau \neq I d$, the local Hamiltonians $\mathbf{H}_{\mathbf{B}_{L}(\mathbf{x})}$ and $\mathbf{H}_{\mathbf{B}_{L}(\mathbf{y})}$ have identical spectra, no matter how far apart the centers $\mathbf{x}$ and $\mathbf{y}$ are. This renders impossible any kind of EVC bounds for such pairs of operators in the course of the localization analysis. In fact, this difficulty is still present even if $\mathbf{U}$ is not permutation symmetric, for the probabilistic analysis of eigenvalue concentration relies upon the external random potential $\mathbf{V}(\mathbf{x} ; \omega)=\sum_{j} V\left(x_{j} ; \omega\right)$, which still is permutation symmetric.

On the other hand, this technical problem is merely an artefact of the language of distinguishable particles; in a system with quantum symmetry (i.e., with Bose-Einstein or Fermi-Dirac quantum statistics), the configuration vertices $\mathbf{y}$ and $\mathbf{x}=\tau(\mathbf{y})$ are simply identical. In a properly defined configuration space of $N$ indistinguishable (bosonic or fermionic) particles (cf. Section 4), $\rho_{S}$ is a natural distance.

Definition 5. Let $\mathbf{x} \in \mathscr{Z}^{N}$ and consider an index subset $\mathscr{J} \subset[[1, N]]$ with $1 \leq|\mathscr{F}|=n<N$. A subconfiguration of $\mathbf{x}$ associated with $\mathscr{J}$ is the pair $\left(\mathbf{x}^{\prime}, \mathscr{J}\right)$ where the vector $\mathbf{x}^{\prime} \in \mathscr{Z}^{N}$ has the components $x_{i}^{\prime}=x_{j_{i}}, i \in[[1, n]]$. Such a subconfiguration will be denoted by $\mathbf{x}_{\mathscr{J}}$. The complement of a subconfiguration $\mathbf{x}_{\mathscr{f}}$ is the subconfiguration $\mathbf{x}_{\mathscr{J}^{c}}$ associated with the complementary index subset $\mathscr{J}^{c}:=[[1, N]] \backslash \mathscr{J}$.

By a slight abuse of notation, we will identify a subconfiguration $\mathbf{x}_{\mathscr{J}}=\left(\mathbf{x}^{\prime}, \mathscr{J}\right)$ with the vector $\mathbf{x}^{\prime}$. With $\mathscr{J}$ clearly identified (this will always be the case in our arguments), it should not lead to any ambiguity, while making notation simpler. 
Definition 6. (a) Let $N \geq 2$ and consider the set of all $N$-particle configurations $\mathscr{Z}^{N}$. For each $j \in[[1, N]]$ the coordinate projection $\Pi_{j}: \mathscr{Z}^{N} \rightarrow \mathscr{Z}$ onto the coordinate space of the $j$ th particle is the following mapping:

$$
\Pi_{j}:\left(x_{1}, \ldots, x_{N}\right) \longmapsto x_{j} .
$$

(b) The support $\Pi \mathbf{x}$ of a configuration $\mathbf{x} \in \mathscr{Z}^{N}, N \geq 1$, is the following set:

$$
\Pi \mathbf{x}:=\bigcup_{j=1}^{N} \Pi_{j} \mathbf{x}=\left\{x_{1}, \ldots, x_{N}\right\} .
$$

Similarly, the support of a subconfiguration $\mathbf{x}_{\mathscr{f}}$ is defined by $\Pi \mathbf{x}_{\mathscr{g}}:=\bigcup_{j \in \mathscr{f}}\left\{\Pi_{j} \mathbf{x}\right\}=\bigcup_{j \in \mathcal{F}}\left\{x_{j}\right\}$.

(c) Given an index subset $\mathscr{J} \subset[[1, N]]$, the projection $\Pi_{\mathscr{J}}: \mathscr{Z}^{N} \rightarrow \mathscr{Z}$ is defined as follows:

$$
\Pi_{\mathscr{J}} \mathbf{x}= \begin{cases}\Pi \mathbf{x}_{\mathcal{J}}, & \text { if } \mathscr{J} \neq \varnothing \\ \varnothing, & \text { otherwise }\end{cases}
$$

For a subset $\mathbf{B} \subset \mathscr{Z}^{N}$ its support $\Pi \mathbf{B}$ is defined by

$$
\Pi \mathbf{B}:=\bigcup_{j=1}^{N} \Pi_{j} \mathbf{B} \subset \mathscr{Z} .
$$

\subsection{Weakly Separated Ball}

Definition 7. A ball $\mathbf{B}_{L}(\mathbf{x})$ is weakly separated from $\mathbf{B}_{L}(\mathbf{y})$ if there exists a bounded subset $Q \subset \mathscr{Z}$ in the 1-particle configuration space, of diameter $R \leq 2 N L$, and the index subsets $\mathscr{J}_{1}, \mathscr{J}_{2} \subset[[1, N]]$ such that $\left|\mathscr{F}_{1}\right|>\left|\mathscr{J}_{2}\right|$ (possibly, with $\mathscr{J}_{2}=\varnothing$ ) and

$$
\begin{gathered}
\left(\Pi_{\mathscr{F}_{1}} \mathbf{B}_{L}(\mathbf{x}) \cup \Pi_{\mathscr{f}_{2}} \mathbf{B}_{L}(\mathbf{y})\right) \subseteq Q, \\
\left(\Pi_{\mathscr{f}_{1}} \mathbf{B}_{L}(\mathbf{x}) \cup \Pi_{\mathscr{f}_{2}} \mathbf{B}_{L}(\mathbf{y})\right) \cap Q=\varnothing .
\end{gathered}
$$

A pair of balls $\left(\mathbf{B}_{L}(\mathbf{x}), \mathbf{B}_{L}(\mathbf{y})\right)$ is weakly separated if at least one of the balls is weakly separated from the other.

The physical meaning of the weak separation is that in a certain region of the one-particle configuration space the presence of particles from $\mathbf{x}$ is more important than that of the particles from $y$. As a result, some local fluctuations of the random potential $V(\cdot ; \omega)$ have a stronger influence on $\mathbf{H}_{\mathbf{B}_{L}(\mathbf{x})}$ than on $\mathbf{H}_{\mathbf{B}_{L}(\mathbf{y})}$.

In fact, (29) shows, as does the application to the proof of Lemma 9 below, that one can take the minimal set $Q$ satisfying (29), that is, $Q=\Pi_{J_{1}} \mathbf{B}_{L}(\mathbf{x}) \cup \Pi_{J_{2}} \mathbf{B}_{L}(\mathbf{y})$. However, we keep a more general form, since some variations may prove useful in particular models and applications.

Lemma 8. Any pair of $N$-particle balls $\mathbf{B}_{L}(\mathbf{x}), \mathbf{B}_{L}(\mathbf{y})$ with $\boldsymbol{\rho}_{S}(\mathbf{x}, \mathbf{y})>(4 N-2) L$ is weakly separated.

Proof. Given $\mathbf{x}=\left(x_{1}, \ldots, x_{N}\right)$, consider the collection of 1particle balls $B_{2 L}\left(x_{j}\right), j=1, \ldots, N$, of double radius $2 L$, and call a cluster any connected union of these balls $B_{2 L}\left(x_{j}\right)$ which is minimal, that is, nondecomposable into smaller disjoint connected unions (of the abovementioned balls $B_{2 L}\left(x_{j}\right)$ ).

Let $\boldsymbol{\Gamma}(\mathbf{x})=\left\{\Gamma_{1}, \ldots, \Gamma_{M}\right\}, 1 \leq M \leq N$, be the collection of clusters of the 1-particle balls $B_{2 L}\left(x_{j}\right)$, so that $[[1, N]]=$ $\sqcup_{1 \leq i \leq M} J_{i}, J_{i}=\left\{j: x_{j} \in \Gamma_{i}\right\}$ and $\Gamma_{i}=\bigcup_{j \in J_{i}} B_{2 L}\left(x_{j}\right)$.

Further, denote $Q_{i}=\bigcup_{j \in J_{i}} B_{L}\left(x_{j}\right)$ (this time, we take the radius $L$, not $2 L$ ). The clusters are connected sets, thus

$$
\operatorname{diam} Q_{i} \leq \sum_{j \in J_{i}} \operatorname{diam} B_{2 L}\left(x_{j}\right) \leq 4 N L
$$

Note that $B_{2 L}\left(x_{j}\right) \cap B_{2 L}\left(x_{k}\right) \neq \varnothing \Leftrightarrow d\left(x_{j}, x_{k}\right) \leq 4 L$; thus we have the following bounds:

$$
\forall i=1, \ldots, M \quad \operatorname{diam}\left\{x_{j}, j \in J_{i}\right\} \leq 4 L(N-1),
$$

and if $y \in \Gamma_{i}$, then $\min _{j \in J_{i}} d\left(y, x_{j}\right) \leq 2 L$, yielding

$$
\begin{aligned}
\max _{j \in J_{i}} d\left(y, x_{j}\right) & \leq 2 L+\operatorname{diam}\left\{x_{j}, j \in J_{i}\right\} \\
& \leq 2 L+4 L(N-1) \\
& =(4 N-2) L .
\end{aligned}
$$

Introduce the occupation numbers of the sets $\Gamma_{i}$ for configurations $\mathbf{x}$ and $\mathbf{y}$ :

$$
\begin{array}{ll}
n_{i}(\mathbf{x})=\operatorname{card}\left(\Pi \mathbf{x} \cap \Gamma_{i}\right), & i \in[[1, M]], \\
n_{i}(\mathbf{y})=\operatorname{card}\left(\Pi \mathbf{y} \cap \Gamma_{i}\right), & i \in[[1, M]] .
\end{array}
$$

It follows immediately from the definition of $\Gamma_{i} \in \Gamma(\mathbf{x})$ that $n_{i}(\mathbf{x})=\left|J_{i}\right|, i=1, \ldots, M$; thus $\sum_{i} n_{i}(\mathbf{x})=N$, while for the configuration $\mathbf{y}$ we only have, in general, that $0 \leq \sum_{i} n_{i}(\mathbf{y}) \leq$ $N$.

There can be two possible situations.

(I) For all $i \in[[1, M]]$ we have $n_{i}(\mathbf{x})=n_{i}(\mathbf{y})$. Then by (32), there exists a permutation $\tau \in \mathbb{S}_{N}$ such that, for all $j \in[[1, N]]$, the following holds:

$$
d\left(x_{\tau(j)}, y_{j}\right) \leq(4 N-2) L
$$

yielding, by (22)-(23), the following:

$$
\begin{aligned}
\boldsymbol{\rho}_{S}(\mathbf{x}, \mathbf{y}) & \leq \boldsymbol{\rho}(\tau(\mathbf{x}), \mathbf{y})=\max _{1 \leq j \leq N} d\left(x_{\tau(j)}, y_{j}\right) \\
& \leq 4 N L-2 L .
\end{aligned}
$$

If $\boldsymbol{\rho}_{S}(\mathbf{x}, \mathbf{y})>(4 N-2) L$, then the occupation numbers $n_{i}(\mathbf{x}), n_{i}(\mathbf{y})$ cannot be all identical, so this situation is impossible under the hypotheses of the lemma.

(II) For some $i \in[[1, M]], n_{i}(\mathbf{x}) \neq n_{i}(\mathbf{y})$. As was already mentioned, consider the following:

$$
\sum_{i=1}\left(n_{i}(\mathbf{x})-n_{i}(\mathbf{y})\right)=N-\sum_{i=1} n_{i}(\mathbf{y}) \geq 0
$$


Since not all the summands $\left(n_{i}(\mathbf{x})-n_{i}(\mathbf{y})\right)$ vanish and the LHS of (36) is nonnegative, there is some $j_{\circ} \epsilon$ $[[1, M]]$ such that $n_{j_{0}}(\mathbf{x})-n_{j_{0}}(\mathbf{y})>0$.

Setting $Q=Q_{j_{0}}, \mathscr{J}_{1}=J_{j_{0}}$, and $\mathscr{J}_{2}=\left\{j: y_{j} \in Q_{j_{0}}\right.$, we conclude that the conditions (29) are fulfilled and $\left|\mathscr{J}_{1}\right|-\left|\mathscr{J}_{2}\right| \geq n_{j_{0}}(\mathbf{x})-n_{j_{0}}(\mathbf{y})>0$.

\section{Eigenvalue Concentration Bound for the Pairs of Distant Balls}

\subsection{EVC Bound for Weakly Separated Balls}

Lemma 9. Let $V: \mathscr{Z} \times \Omega \rightarrow \mathbb{R}$ be a random field satisfying the condition (RCM). Let $\mathbf{x}, \mathbf{y} \in \mathscr{Z}^{N}$ be two configurations such that the balls $\mathbf{B}_{L}(\mathbf{x}), \mathbf{B}_{L}(\mathbf{y})$ are weakly separated. Consider the operators $\mathbf{H}_{\mathbf{B}_{L^{\prime}}(\mathbf{y})}(\omega), \mathbf{H}_{\mathbf{B}_{L^{\prime \prime}}(\mathbf{y})}(\omega)$, with $L^{\prime}, L^{\prime \prime} \leq L$. Then for any $s>0$ the following bound holds for the spectra $\Sigma_{\mathbf{x}}=\sigma\left(\mathbf{H}_{\mathbf{B}_{L^{\prime}}(\mathbf{x})}\right)$, $\Sigma_{\mathbf{y}}=\sigma\left(\mathbf{H}_{\mathbf{B}_{L^{\prime \prime}}(\mathbf{y})}\right)$ of these operators:

$$
\begin{aligned}
& \mathbb{P}\left\{\operatorname{dist}\left(\Sigma_{\mathbf{x}}, \Sigma_{\mathbf{y}}\right) \leq s\right\} \\
& \leq\left|\mathbf{B}_{L}(\mathbf{x})\right|\left|\mathbf{B}_{L}(\mathbf{y})\right| C^{\prime} L^{A^{\prime}}(2 s)^{b^{\prime}}+C^{\prime \prime} L^{A^{\prime \prime}}(2 s)^{b^{\prime \prime}} .
\end{aligned}
$$

Proof. Let $Q$ be a set satisfying the conditions (29) for some $\mathscr{J}_{1}, \mathscr{J}_{2} \subset[[1, N]]$ with $\left|\mathscr{F}_{1}\right|=: n_{1}>n_{2}:=\left|\mathscr{J}_{2}\right|$. Consider the sample mean $\xi=\xi_{Q}$ of $V$ over $Q$ and the fluctuations $\left\{\eta_{x}, x \in Q\right\}$ defined in (12).

The operators $\mathbf{H}_{\mathbf{B}_{L^{\prime}}(\mathbf{x})}(\omega), \mathbf{H}_{\mathbf{B}_{L^{\prime \prime}}(\mathbf{y})}(\omega)$ can be represented as follows:

$$
\begin{aligned}
& \mathbf{H}_{\mathbf{B}_{L^{\prime}}(\mathbf{x})}(\omega)=n_{1} \xi(\omega) \mathbf{1}+\mathbf{A}(\omega), \\
& \mathbf{H}_{\mathbf{B}_{L^{\prime \prime}}(\mathbf{y})}(\omega)=n_{2} \xi(\omega) \mathbf{1}+\mathbf{B}(\omega),
\end{aligned}
$$

where the operators $\mathbf{A}(\omega)$ and $\mathbf{B}(\omega)$ are $\mathfrak{F}_{\mathrm{Q}}$-measurable. Specifically, let $\mathscr{F}_{1}^{c}=[[1, N]] \backslash \mathscr{F}_{1}, \mathscr{F}_{2}^{c}=[[1, N]] \backslash \mathscr{J}_{2}$, and

$$
\begin{aligned}
& \mathbf{A}(\omega)=\Delta+\mathbf{U}_{\mathbf{B}_{L^{\prime}}(\mathbf{x})}+\sum_{j \in \mathscr{F}_{1}^{c}} V\left(x_{j} ; \omega\right)+\sum_{j \in \mathscr{F}_{1}} \eta_{x_{j}}(\omega), \\
& \mathbf{B}(\omega)=\Delta+\mathbf{U}_{\mathbf{B}_{L^{\prime \prime}}(\mathbf{y})}+\sum_{j \in \mathscr{F}_{2}^{c}} V\left(y_{j} ; \omega\right)+\sum_{j \in \mathscr{F}_{2}} \eta_{y_{j}}(\omega) .
\end{aligned}
$$

Now (38) follows from the identities (cf. (12)) as follows:

$$
\begin{array}{ll}
V\left(x_{j} ; \omega\right)=\xi(\omega)+\eta_{x_{j}}(\omega), & j \in \mathscr{J}_{1}, \\
V\left(y_{j} ; \omega\right)=\xi(\omega)+\eta_{y_{j}}(\omega), & j \in \mathscr{J}_{2},
\end{array}
$$

since $\Pi_{\mathscr{F}_{1}} \mathbf{B}_{L^{\prime}}(\mathbf{x}), \Pi_{\mathscr{F}_{2}} \mathbf{B}_{L^{\prime \prime}}(\mathbf{y}) \subset Q,\left|\mathscr{J}_{1}\right|=n_{1},\left|\mathscr{F}_{2}\right|=n_{2}$.

Let $\left\{\lambda_{1}, \ldots, \lambda_{M^{\prime}}\right\}$ and $\left\{\mu_{1}, \ldots, \mu_{M^{\prime \prime}}\right\}$, with $M^{\prime}=\left|\mathbf{B}_{L^{\prime}}(\mathbf{x})\right|$, $M^{\prime \prime}=\left|\mathbf{B}_{L^{\prime \prime}}(\mathbf{y})\right|$, be the sets of eigenvalues of $\mathbf{H}_{\mathbf{B}_{L^{\prime}}(\mathbf{x})}$ and of $\mathbf{H}_{\mathbf{B}_{L^{\prime \prime}}(\mathbf{y})}$, counting multiplicities. By (38), these eigenvalues can be represented as follows:

$$
\begin{aligned}
& \lambda_{j}(\omega)=n_{1} \xi(\omega)+\lambda_{j}^{(0)}(\omega), \\
& \mu_{j}(\omega)=n_{2} \xi(\omega)+\mu_{j}^{(0)}(\omega),
\end{aligned}
$$

where the random variables $\lambda_{j}^{(0)}(\omega)$ and $\mu_{j}^{(0)}(\omega)$ are $\mathfrak{F}_{Q^{-}}$ measurable. Therefore, consider

$$
\lambda_{i}(\omega)-\mu_{j}(\omega)=\left(n_{1}-n_{2}\right) \xi(\omega)+\left(\lambda_{j}^{(0)}(\omega)-\mu_{j}^{(0)}(\omega)\right),
$$

with $n_{1}-n_{2} \geq 1$, by our assumption. Further, we can write

$$
\begin{aligned}
\mathbb{P}\left\{\operatorname{dist}\left(\Sigma_{\mathbf{x}}, \Sigma_{\mathbf{y}}\right) \leq s\right\} & =\mathbb{P}\left\{\exists i, j:\left|\lambda_{i}-\mu_{j}\right| \leq s\right\} \\
& \leq \sum_{i=1}^{M^{\prime}} \sum_{j=1}^{M^{\prime \prime}} \mathbb{E}\left[\mathbb{P}\left\{\left|\lambda_{i}-\mu_{j}\right| \leq s \mid \mathfrak{F}_{\mathrm{Q}}\right\}\right] .
\end{aligned}
$$

Note that for all $i$ and $j$ we have

$$
\begin{aligned}
& \mathbb{P}\left\{\left|\lambda_{i}-\mu_{j}\right| \leq s \mid \mathfrak{\Im}_{Q}\right\} \\
& \quad=\mathbb{P}\left\{\left|\left(n_{1}-n_{2}\right) \xi+\lambda_{i}^{(0)}-\mu_{j}^{(0)}\right| \leq s \mid \mathfrak{F}_{\mathrm{Q}}\right\} \\
& \quad \leq \sup _{t \in \mathbb{R}} \mathbb{P}\left\{|\xi-t| \leq\left(n_{1}-n_{2}\right)^{-1} s \mid \mathfrak{F}_{\mathrm{Q}}\right\} \\
& \quad \leq \sup _{t \in \mathbb{R}}\left(F_{\xi}\left(t+s \mid \mathfrak{F}_{\mathrm{Q}}\right)-F_{\xi}\left(t-s \mid \mathfrak{F}_{\mathrm{Q}}\right)\right)
\end{aligned}
$$

(we used $\left.\left(n_{1}-n_{2}\right)^{-1} \leq 1\right)$. Consider the event

$$
\begin{aligned}
& \mathscr{E}_{L} \\
& =\left\{\sup _{t \in \mathbb{R}}\left|F_{\xi}\left(t+2 s \mid \mathfrak{\mho}_{\mathrm{Q}}\right)-F_{\xi}\left(t \mid \mathfrak{\Im}_{\mathrm{Q}}\right)\right| \geq C^{\prime} L^{A^{\prime}}(2 s)^{b^{\prime}}\right\} .
\end{aligned}
$$

By (RCM) (cf. (14)), $\mathbb{P}\left\{\mathscr{E}_{L}\right\} \leq C^{\prime \prime} L^{A^{\prime \prime}}(2 s)^{b^{\prime \prime}}$. Therefore,

$$
\begin{aligned}
\mathbb{P}\{\operatorname{dist} & \left.\left(\Sigma_{\mathbf{x}}, \Sigma_{\mathbf{y}}\right) \leq s\right\} \\
= & \mathbb{E}\left[\mathbb{P}\left\{\operatorname{dist}\left(\Sigma_{\mathbf{x}}, \Sigma_{\mathbf{y}}\right) \leq s \mid \mathfrak{F}_{\mathbf{Q}}\right\}\right] \\
\leq & \mathbb{E}\left[\mathbf{1}_{\mathscr{C}_{L}} \mathbb{P}\left\{\operatorname{dist}\left(\Sigma_{\mathbf{x}}, \Sigma_{\mathbf{y}}\right) \leq s \mid \mathfrak{F}_{\mathbf{Q}}\right\}\right]+\mathbb{P}\left\{\mathscr{E}_{L}\right\} \\
\leq & \left|\mathbf{B}_{L^{\prime \prime}}(\mathbf{x})\right| \cdot\left|\mathbf{B}_{L^{\prime \prime}}(\mathbf{y})\right| C^{\prime} L^{A^{\prime}}(2 s)^{b^{\prime}}+C^{\prime \prime} L^{A^{\prime \prime}}(2 s)^{b^{\prime \prime}} .
\end{aligned}
$$

3.2. Proof of Theorem 3. By hypothesis, we have $\boldsymbol{\rho}_{S}(\mathbf{x}, \mathbf{y})>$ $(4 N-2) L$, thus, by Lemma 8 , the balls $\mathbf{B}_{L^{\prime}}(\mathbf{x})$ and $\mathbf{B}_{L^{\prime \prime}}(\mathbf{y})$ are weakly separated, and the claim follows from Lemma 9.

\section{EVC Bounds for the Particle Systems with Quantum Symmetry}

The main EVC bound, established for the Hamiltonian $\mathbf{H}(\omega)$ in the entire Hilbert space $\ell^{2}\left(\mathscr{Z}^{N}\right)$, implies a lower bound on the spectral spacings for the restrictions of $\mathbf{H}(\omega)$ to the subspaces of symmetric and of antisymmetric functions, that 
is, for the bosonic and fermionic $N$-particle Hamiltonians. For the convenience of further references, we introduce below required notations and objects.

A technically convenient alternative to restricting $\mathbf{H}$ to a subspace of $( \pm)$-symmetric functions $\Psi:(\mathscr{Z})^{N} \rightarrow \mathbb{C}$ consists in representing these subspaces as the spaces of square-summable functions on a reduced, quotient graph. Such reduction is the most straight-forward in the case where $\mathscr{Z}=\mathbb{Z}^{1}$. In this case, consider the following.

(i) The subspace of all antisymmetric square-summable functions $\Psi:(\mathbb{Z})^{N} \rightarrow \mathbb{C}$ in the Hilbert space $\mathscr{H}^{(N)}=$ $\ell^{2}\left(\mathbb{Z}^{N}\right)$ is canonically isomorphic to the space $\ell^{2}\left(\mathbb{Z}_{>}^{N}\right)$ with

$$
\mathbb{Z}_{>}^{N}:=\left\{\mathbf{x} \in \mathbb{Z}^{N}: x_{N}>x_{N-1}>\cdots>x_{1}\right\} .
$$

Up to a constant factor of $(N !)^{1 / 2}$, the canonical isomorphism is given by the restriction $\Psi \mapsto$ $\left(\mathbf{1}_{\mathbb{Z}_{\geq}^{N}} \Psi\right)\left\lceil_{\mathbb{Z}_{\geq}^{N}}\right.$

(ii) Similarly, the subspace of symmetric functions $\Psi$ : $(\mathbb{Z})^{N} \rightarrow \mathbb{C}$ in $\mathscr{H}^{(N)}=\ell^{2}\left(\mathbb{Z}^{N}\right)$ is canonically isomorphic to the weighted space $\ell^{2}\left(\mathbb{Z}_{\geq}^{N}, \mathfrak{t}\right)$ with

$$
\mathbb{Z}_{\geq}^{N}:=\left\{\mathbf{x} \in \mathbb{Z}^{N}: x_{N} \geq x_{N-1} \geq \cdots \geq x_{1}\right\}
$$

and the combinatorial weight $\mathfrak{f}$ taking into account the multiplicity of a point $u \in \mathscr{Z}$ in a particle configuration $\left(x_{1}, \ldots, x_{N}\right)$; we omit the precise formula, for it is more natural to start with a self-consistent representation of a system of indistinguishable particles, without referring to an artificial numeration of their positions.

In a more general case, there are alternative constructions of the reduced graph.

4.1. Fermionic Graph. The standard construction of a symmetric power of an arbitrary locally finite graph $\mathscr{Z}$ is most suitable for the fermionic systems. Recall that, by definition, for $N \geq 2$, the $N$ th symmetric power of the graph $(\mathscr{Z}, \mathscr{E})$ is the graph with the vertex set formed by the $N$-tuplets $\mathbf{x}=$ $\left(x_{1}, \ldots, x_{N}\right)$ with $x_{j} \in \mathscr{E}, j \in[[1, N]]$ and $\operatorname{card}\left\{x_{1}, \ldots, x_{N}\right\}=$ $N$, that is, without duplicate positions in the graph $\mathscr{Z}$. Clearly, this is a subset of the edge set of the product graph $\mathscr{Z}^{N}$ considered before. The edges are those inherited from $\mathscr{E}^{(N)}$ : $(\mathbf{x}, \mathbf{y})$ is an edge if and only if $\sum_{j} d_{\mathscr{E}}\left(x_{j}, y_{j}\right)=1$, or, equivalently, if for some pairwise distinct $z_{2}, \ldots, z_{N}$ are as follows:

$$
\begin{gathered}
\mathbf{x}=\left\{x, z_{2}, \ldots, z_{N}\right\}, \quad \mathbf{y}=\left\{y, z_{2}, \ldots, z_{N}\right\}, \\
\operatorname{card}\left\{x, y, z_{2}, \ldots, z_{N}\right\}=N+2 .
\end{gathered}
$$

As before, these relations can be interpreted in the following way: the configuration of $N$ indistinguishable particles $\mathbf{y}$ is obtained by moving exactly one particle from the configuration $\mathbf{x}$ (without duplicate positions) to one of its nearest unoccupied neighbors in $\mathscr{Z}$.
4.2. Representation by the Occupation Numbers. An alternative construction, which we present first in the fermionic case, is easily adapted to the bosonic systems.

A configuration of $N$ indistinguishable particles, $N \geq 1$, is uniquely determined by an "NN-decorated" subset of $\mathscr{Z}$ formed by all particle positions along with their respective multiplicities. (In other words, we consider formal finite linear combinations of vertices from $\mathscr{Z}$ with nonnegative integer coefficients). Specifically, introduce the functions $\mathbf{n}_{\mathbf{x}}$ : $\mathscr{Z} \rightarrow \mathbb{N}$, associated with the indistinguishable particle configurations $\mathbf{x}$, with the value $\mathbf{n}_{\mathbf{x}}(u)$ interpreted as the number of particles at $u \in \mathscr{Z}$ from the configuration $\mathbf{x}$. We require the following:

$$
\sum_{u \in \mathscr{Z}} \mathbf{n}_{\mathbf{x}}(u)=N
$$

In the fermionic case, we require in addition the following:

$$
\mathbf{n}_{\mathbf{x}}: \mathscr{Z} \longrightarrow\{0,1\},
$$

which is a tantamount to assuming the particle positions to be pairwise distinct. To define the required graph structure in the set of the occupation number functions, call a pair $\left(\mathbf{n}^{\prime}, \mathbf{n}^{\prime \prime}\right)$ an edge if and only if

$$
\sum_{u \in \mathscr{X}}\left|\mathbf{n}^{\prime}(u)-\mathbf{n}^{\prime \prime}(u)\right|=2, \quad \operatorname{diam} \operatorname{supp}\left(\mathbf{n}^{\prime}-\mathbf{n}^{\prime \prime}\right)=1 .
$$

In other words, $\operatorname{supp}\left(\mathbf{n}^{\prime}-\mathbf{n}^{\prime \prime}\right)=\{x, y\}$, with $d(x, y)=1$, so $(x, y) \in \mathscr{E}$ is an edge in $\mathscr{Z}$.

For example, with $\mathscr{Z}=\mathbb{Z}$, the 2-particle configurations $\mathbf{x}=(0,1)$ and $\mathbf{y}=(0,2)$, with occupation numbers $\mathbf{n}_{\mathbf{x}}=$ $\mathbf{1}_{\{0\}}+\mathbf{1}_{\{1\}}, \mathbf{n}_{\mathbf{y}}=\mathbf{1}_{\{0\}}+\mathbf{1}_{\{2\}}$, form an edge, since

$$
\mathbf{n}_{\mathbf{x}}-\mathbf{n}_{\mathbf{y}}=\mathbf{1}_{\{1\}}-\mathbf{1}_{\{2\}} ;
$$

thus

$$
\begin{gathered}
\operatorname{diam} \operatorname{supp}\left(\mathbf{n}_{\mathbf{x}}-\mathbf{n}_{\mathbf{y}}\right)=\operatorname{diam}\{1,2\}=1, \\
\sum_{u \in \mathbb{Z}}\left|\mathbf{n}_{\mathbf{x}}(u)-\mathbf{n}_{\mathbf{y}}(u)\right|=\sum_{u \in\{1,2\}}\left|\mathbf{n}_{\mathbf{x}}(u)-\mathbf{n}_{\mathbf{y}}(u)\right|=2 .
\end{gathered}
$$

The vertex set of the $N$-fermionic graph over $\mathscr{Z}$ will be denoted by $\mathscr{Z}_{-}^{N}$.

4.3. Bosonic Graph. Consider the integer-valued functions $\mathbf{n}_{\mathbf{x}}: \mathscr{Z} \rightarrow[[0, N]]$ obeying

$$
\sum_{u \in \mathscr{X}} \mathbf{n}_{\mathbf{x}}(u)=N
$$

This allows for duplicate positions; for example, one can have $\mathbf{n}_{\mathbf{x}}=N \mathbf{1}_{x}$, interpreted as the configuration $\mathbf{x}=(x, x, \ldots, x)$, with $N$-particles occupying the same position $x \in \mathscr{Z}$. The edges $\left(\mathbf{n}^{\prime}, \mathbf{n}^{\prime \prime}\right)$ are still defined by the following constraints:

$$
\sum_{u \in \mathscr{Z}}\left|\mathbf{n}^{\prime}(u)-\mathbf{n}^{\prime \prime}(u)\right|=2, \quad \operatorname{diam} \operatorname{supp}\left(\mathbf{n}^{\prime}-\mathbf{n}^{\prime \prime}\right)=1,
$$


meaning, as before, that exactly one particle from $\mathbf{x}$ is moved to one of its neighboring positions. Indeed, exactly two terms in the above sum are nonzero integers, with sum $=2$, so both are equal to 1 ; by the conservation law (55), one of the differences under the absolute value sign is +1 and the other one -1 .

For example, $\mathbf{x}=(a, a, b)$ and $\mathbf{y}=(a, b, b)$ with $d(a, b)=1$ form an edge:

$$
\mathbf{n}_{\mathbf{x}}=2 \mathbf{1}_{\{a\}}+\mathbf{1}_{\{b\}}, \quad \mathbf{n}_{\mathbf{y}}=\mathbf{1}_{\{a\}}+2 \mathbf{1}_{\{b\}},
$$

so $\mathbf{n}_{\mathbf{x}}-\mathbf{n}_{\mathbf{y}}=\mathbf{1}_{\{a\}}-\mathbf{1}_{\{b\}}$, and we have

$$
\begin{gathered}
\operatorname{supp}\left(\mathbf{n}_{\mathbf{x}}-\mathbf{n}_{\mathbf{y}}\right)=\{a, b\}, \quad \operatorname{diam} \operatorname{supp}\left(\mathbf{n}_{\mathbf{x}}-\mathbf{n}_{\mathbf{y}}\right)=1, \\
\sum_{u \in \mathscr{Z}}\left|\mathbf{n}_{\mathbf{x}}(u)-\mathbf{n}_{\mathbf{y}}(u)\right|=\sum_{u \in\{a, b\}}\left|\mathbf{n}_{\mathbf{x}}(u)-\mathbf{n}_{\mathbf{y}}(u)\right|=2 .
\end{gathered}
$$

The vertex set of the $N$-bosonic graph over $\mathscr{Z}$ will be denoted by $\mathscr{Z}_{+}^{N}$.

4.4. Self-Consistent Representation of Bosonic and Fermonic Hamiltonians. The following formula gives an equivalent form of the restriction of the $N$-particle Hamiltonian to the subspace of symmetric (+) or antisymmetric (-) functions in $\ell^{2}(\mathscr{Z})^{N}$, without using any specific order/numeration of the particle positions:

$$
\mathbf{H}_{ \pm}^{(N)}(\omega)=\Delta_{\mathscr{Z}_{ \pm}^{N}}+g \mathbf{V}(\omega)+\mathbf{U}
$$

where $\mathbf{V}(\omega)$ is the operator of multiplication by a random function,

$$
\mathbf{V}(\omega): \mathbf{x} \longmapsto \sum_{x \in \mathbf{x}} V(x ; \omega), \quad \mathbf{x}=\left(x_{1}, \ldots, x_{N}\right),
$$

and $\mathbf{U}$ is the operator of multiplication by the following nonrandom interaction potential:

$$
\mathbf{U}: \mathbf{x} \longmapsto \sum_{\substack{x, y \in \mathbf{x}, x \neq y}} U(|x-y|) .
$$

4.5. Balls in the Fermionic Graph. We focus now on the fermionic case, which corresponds to the physical model of $N$ electrons (which are fermions) in the tight binding approximation.

The choice of the metric, defining the notion of a ball in the $N$-particle configuration space, depends upon the analytic techniques used on the localization analysis, and it is of course not unique. We consider the case where the maxdistance is used; then the balls, like in the case where $\mathscr{Z}=\mathbb{Z}^{d}$, can be described as polydisks, or suitable subsets thereof, taking into account the quantum symmetry. Specifically, consider

$$
\mathbf{B}_{L}(\mathbf{x})=\left\{\mathbf{y} \in \mathscr{Z}_{-}^{N}: \boldsymbol{\rho}(\mathbf{x}, \mathbf{y}) \leq L\right\} .
$$

There is no need here to make use of the subscript " $S$ " in $\rho(\cdot, \cdot)$, as we did in the first part of the paper, since the symmetry is now encoded in the very construction of the fermionic graph $\mathscr{Z}_{-}^{N}$.

The following example clearly explains the difference between the fermionic ball $\mathbf{B}_{L}(\mathbf{x})$ and the Cartesian product of its single-particle projections $B_{L}(x) \subset \mathscr{Z}, x \in \mathbf{x}$.

Example 10. Let $N=2, \mathscr{Z}=\mathbb{Z}^{1}, L=1, \mathbf{x}=\{0,1\}$, and $\mathbf{y}=\{0,3\}$. Then

$$
\begin{aligned}
\mathbf{B}_{1}(\mathbf{x}) & =\{\{1,2\},\{0,1\},\{0,2\},\{-1,0\},\{-1,1\},\{-1,2\}\} \\
& \subsetneq[0,2] \times[-1,1] \equiv B_{1}(0) \times B_{1}(1),
\end{aligned}
$$

while

$$
\mathbf{B}_{1}(\mathbf{y})=[-1,1] \times[2,4] \equiv B_{1}(0) \times B_{1}(3) .
$$

\subsection{The EVC Bound}

Theorem 11. Let $V: \mathscr{Z} \times \Omega \rightarrow \mathbb{R}$ be a random field satisfying (RCM). Then for any pair of $N$-particle fermionic Hamiltonians $\mathbf{H}_{\mathbf{B}_{L^{\prime}}\left(\mathbf{u}^{\prime}\right)}^{(N,-)}, \mathbf{H}_{\mathbf{B}_{L^{\prime \prime}}\left(\mathbf{u}^{\prime \prime}\right)}^{(N,-)}, 0 \leq L^{\prime}, L^{\prime \prime} \leq L$, satisfying $\boldsymbol{\rho}_{S}\left(\mathbf{u}^{\prime}, \mathbf{u}^{\prime \prime}\right)>(4 N-2) L$, and any $s>0$ the following bound holds:

$$
\mathbb{P}\left\{\operatorname{dist}\left(\sigma\left(\mathbf{H}_{\mathbf{B}_{L^{\prime}}\left(\mathbf{u}^{\prime}\right)}^{(N,-)}\right), \sigma\left(\mathbf{H}_{\mathbf{B}_{L^{\prime \prime}}\left(\mathbf{u}^{\prime \prime}\right)}^{(N,-)}\right)\right) \leq s\right\}=h_{L}(s)
$$

with

$$
h_{L}(s):=\left|\mathbf{B}_{L^{\prime}}(\mathbf{x})\right| \cdot\left|\mathbf{B}_{L^{\prime \prime}}(\mathbf{y})\right| C^{\prime} L^{A^{\prime}} s^{b^{\prime}}+C^{\prime \prime} L^{A^{\prime \prime}} s^{b^{\prime \prime}} .
$$

The same bound holds true for the pair of $N$-particle bosonic Hamiltonians $\mathbf{H}_{\mathbf{B}_{L^{\prime}}\left(\mathbf{u}^{\prime}\right)}^{(N,+)}$ and $\mathbf{H}_{\mathbf{B}_{L^{\prime \prime}}\left(\mathbf{u}^{\prime \prime}\right)}^{(N,+)}$, except that the volumes of the bosonic balls $\mathbf{B}_{L^{\prime}}(\mathbf{x}), \mathbf{B}_{L^{\prime \prime}}(\mathbf{y})$ may be different from their fermionic counterparts.

Proof. The claim follows from Theorem 3; it can also be proved directly for the Hamiltonians in the fermionic (resp., bosonic) graphs $\mathscr{Z}_{+}^{N}$, repeating the proof of Theorem 3 almost verbatim, with minor notational adaptations.

\section{Conflict of Interests}

The author declares that there is no conflict of interests regarding the publication of this paper.

\section{Acknowledgments}

It is a pleasure to thank Werner Kirsch and the Fern Universität Hagen for their warm hospitality and simulating atmosphere at the PasturFest (May 2013) and Abel Klein, Fumihiko Nakano, Nariyuki Minami, Stanislav A. Molchanov, Günter Stolz, and Ivan Veselić for fruitful discussions.

\section{References}

[1] P. W. Anderson, "Absence of diffusion in certain random lattices," Physical Review, vol. 109, no. 5, pp. 1492-1505, 1958. 
[2] D. M. Basko, I. L. Aleiner, and B. L. Altshuler, "Metal-insulator transition in a weakly interacting many-electron system with localized single-particle states," Annals of Physics, vol. 321, no. 5, pp. 1126-1205, 2005.

[3] I. V. Gornyi, A. D. Mirlin, and D. G. Polyakov, "Interacting electrons in disordered wires: anderson localization and lowT transport," Physical Review Letters, vol. 95, Article ID 206603, 2005.

[4] V. Chulaevsky and Y. Suhov, "Eigenfunctions in a two-particle Anderson tight binding model," Communications in Mathematical Physics, vol. 289, no. 2, pp. 701-723, 2009.

[5] V. Chulaevsky and Y. Suhov, "Multi-particle Anderson localisation: induction on the number of particles," Mathematical Physics, Analysis and Geometry, vol. 12, no. 2, pp. 117-139, 2009.

[6] M. Aizenman and S. Warzel, "Localization bounds for multiparticle systems," Communications in Mathematical Physics, vol. 290, no. 3, pp. 903-934, 2009.

[7] V. Chulaevsky, A. Boutet de Monvel, and Y. Suhov, "Dynamical localization for a multi-particle model with an alloy-type external random potential," Nonlinearity, vol. 24, no. 5, pp. 14511472, 2011.

[8] A. Klein and S. T. Nguyen, "The bootstrap multiscale analysis of the multi-particle Anderson model," Journal of Statistical Physics, vol. 151, no. 5, pp. 938-973, 2013.

[9] A. Klein and S. T. Nguyen, "Bootstrap multiscale analysis and localization for multi-particle continuous Anderson Hamiltonians," 2013, http://arxiv.org/abs/1311.4220.

[10] M. Fauser and S. Warzel, "Multiparticle localization for disordered systems on continuous space via the fractional moment method," http://arxiv.org/abs/1402.5832.

[11] F. Wegner, "Bounds on the density of states in disordered systems," Zeitschrift für Physik B Condensed Matter and Quanta, vol. 44, no. 1-2, pp. 9-15, 1981.

[12] V. Chulaevsky, "On the regularity of the conditional distribution of the sample mean," http://arxiv.org/abs/1304.6913.

[13] J. Fröhlich and T. Spencer, "Absence of diffusion in the Anderson tight binding model for large disorder or low energy," Communications in Mathematical Physics, vol. 88, no. 2, pp. 151184, 1983.

[14] M. Aizenman and S. Molchanov, "Localization at large disorder and at extreme energies: an elementary derivation," Communications in Mathematical Physics, vol. 157, no. 2, pp. 245-278, 1993.

[15] V. Chulaevsky and Y. Suhov, "Wegner bounds for a two-particle tight binding model," Communications in Mathematical Physics, vol. 283, no. 2, pp. 479-489, 2008.

[16] V. Chulaevsky and Y. Suhov, "Anderson localisation for an interacting two-particle quantum system on $\mathbb{Z}$," http://arxiv .org/abs/0705.0657. 


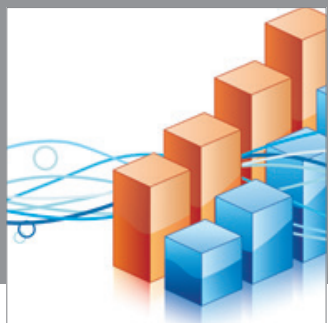

Advances in

Operations Research

mansans

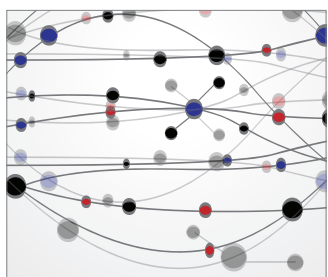

The Scientific World Journal
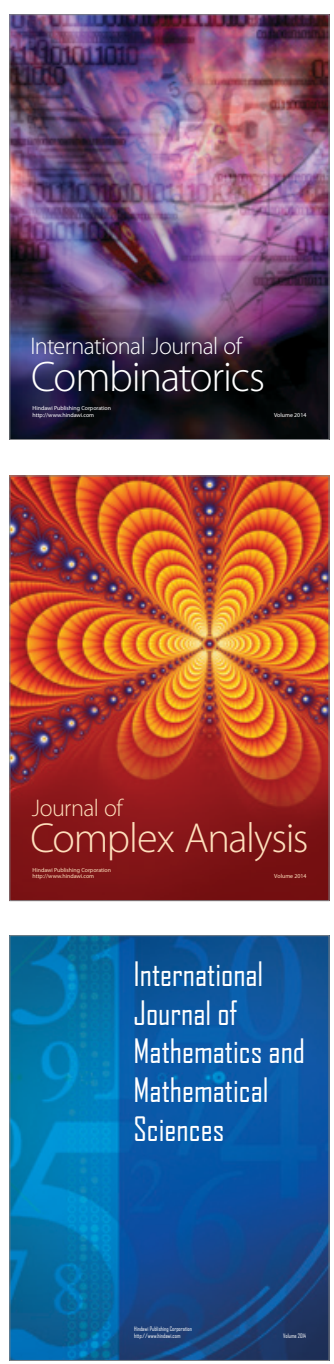
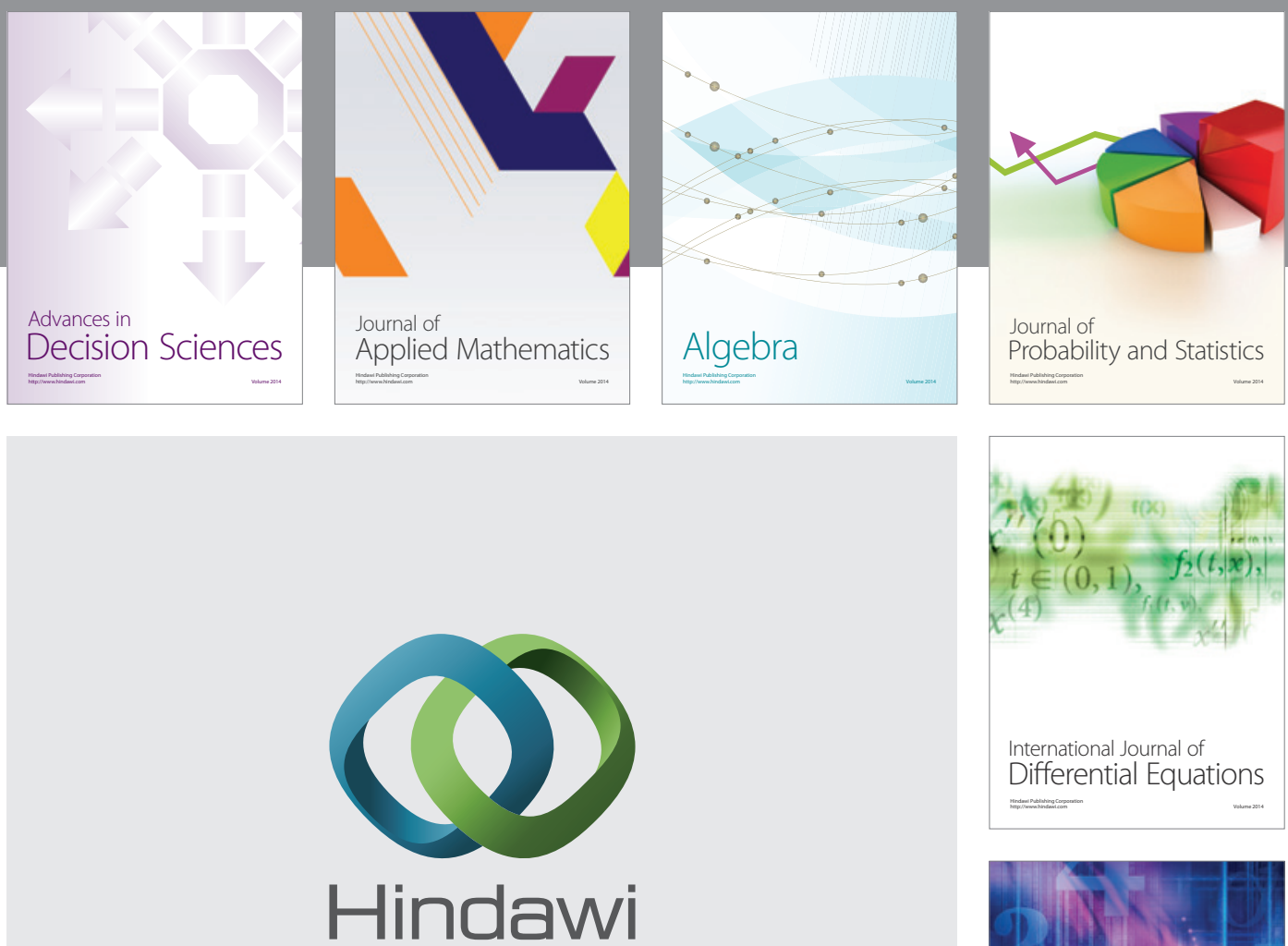

Submit your manuscripts at http://www.hindawi.com
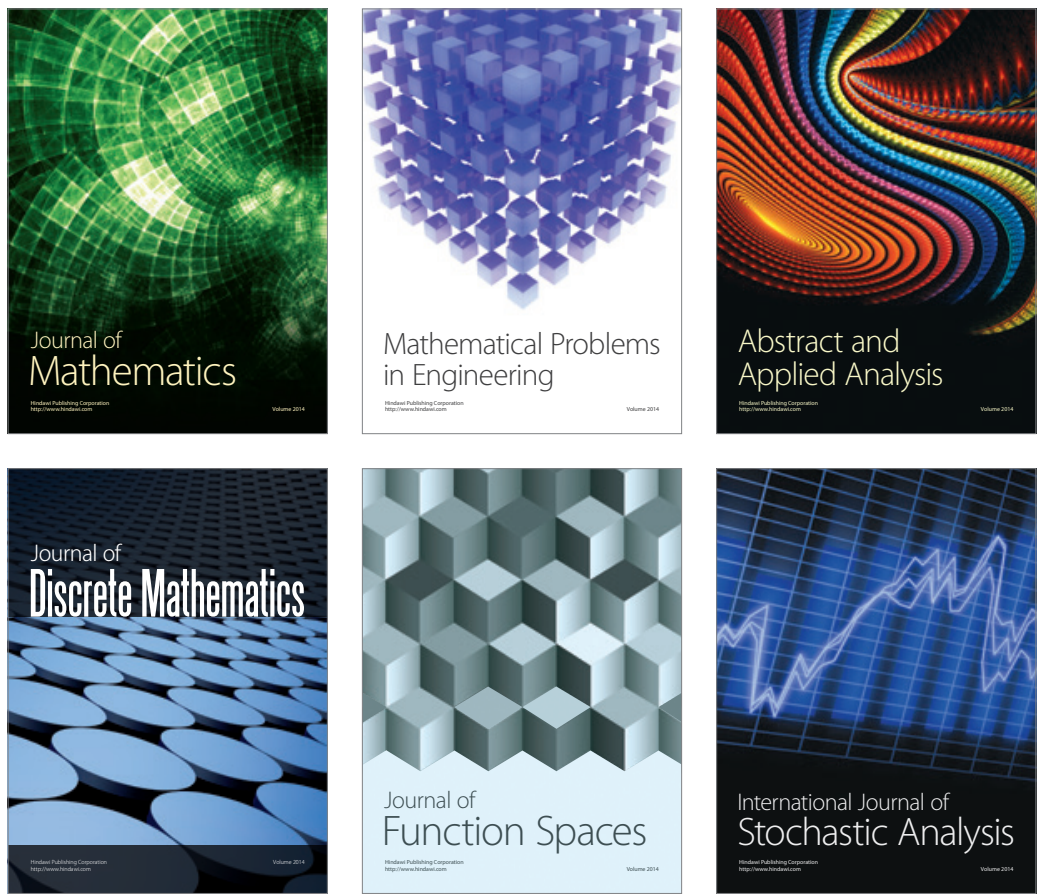

Journal of

Function Spaces

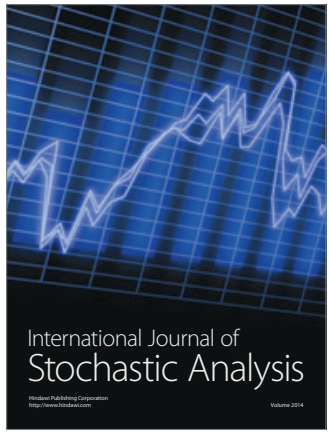

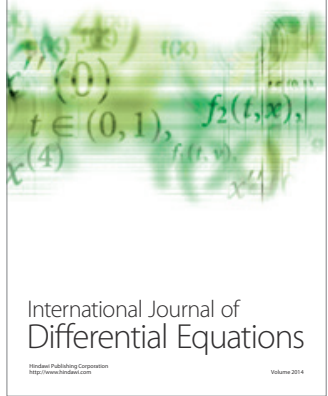
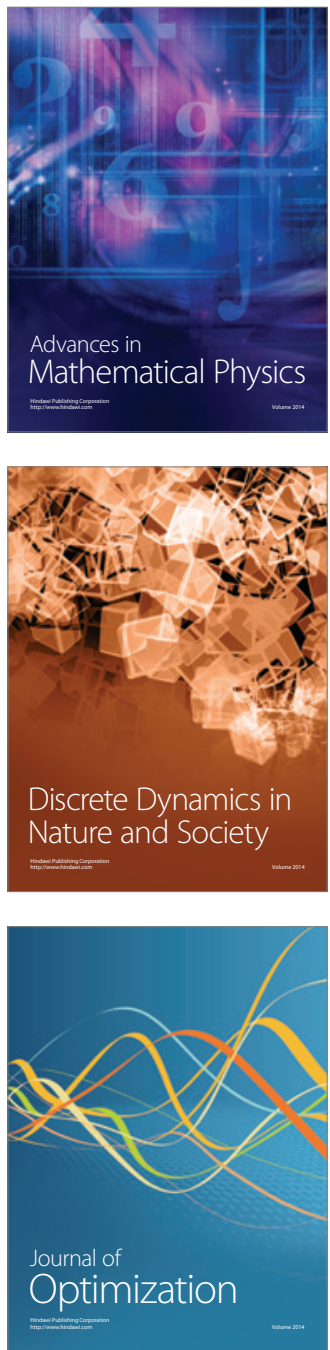